Rev. Elev. Méd. vét. Pays trop., 1974, 27 (4) : 431-436

\title{
Caryotype de Glossina palpalis gambiensis Vanderplank, 1949. Comparaison avec d'autres espèces du groupe palpalis et du groupe morsitans
}

\author{
par J. ITARD (*)
}

\begin{abstract}
RESUME
Glossina palpalis gambiensis possède, comme les autres espèces du sous-genre Nemorhina (Groupe palpalis), quatre autosomes $\left(2 \mathrm{~L}_{1}+2 \mathbf{L}_{2}\right)$ et deux chromosomes sexuels (XX ou $X Y)$. Les especes du sous-genre Glossina (Groupe morsitans), possèdent en outre un jeu de petits chromosomes surnuméraires, hétérochromatiques, dont le nombre varie d'une espèce, ou sous-espèce, à l'autre.

Les euchromosomes $\left(\mathrm{L}_{1}, \mathrm{~L}_{2}, \mathrm{X}\right)$ sont très semblables dans les deux sous-genres. Par contre, le chromosome $Y$, hétérochromatique, est presque acrocentrique chez $G$. $f$. fuscipes et $G$. m. morsitans, alors qu'il est métacentrique chez G.p. gambiensis, G.p. palpalis, G. tachinoides, G. austeni, G. m. submorsitans et $G . m$. centralis.
\end{abstract}

\section{INTRODUCTION}

Depuis 1966, époque où nous avons publié nos premières observations sur les caryotypes de $G$. tachinoides et de $G$. morsitans morsitans (1), nous avons eu l'occasion d'étudier les chromosomes de $G$. fuscipes fuscipes $(3,5)$, de G. austeni $(3,5)$ et de $G$. fusca congolensis (4).

Des études très complètes ont également été effectuées, par SOUTHERN et ses collaborateurs, chez certaines espèces du groupe morsitans $(7,8,9)$ et, plus récemment, chez quelques espèces du groupe palpalis (6).

Enfin nous avons pu, tout dernièrement, étudier les chromosomes de $G$. palpalis gambiensis, espèce que nous élevons dans notre laboratoire depuis juillet 1972 à partir de pupes et d'adultes récoltés en Haute-Volta.

(*) Institut d'Elevage et de Médecine vétérinaire des Pays tropicaux, 10, rue Pierre Curie, 94700 Maisons-Alfort (France).
Il devient ainsi possible de comparer les caryotypes de différentes espèces appartenant soit au sous-genre Nemorhina (groupe palpalis), soit au sous-genre Glossina (groupe morsitans).

\section{CARYOTYPE \\ DE G. PALPALIS GAMBIENSIS VAND., 1949}

Cette espèce possède six chromosomes, dont deux paires d'autosomes et une paire d'hétérosomes ou chromosomes sexuels.

Les autosomes comprennent:

- une paire de grands chromosomes (chromosomes $L_{1}$ ), submétacentriques, avec une constriction secondaire sur le bras le plus long;

- une paire de chromosomes nettement plus courts (chromosomes $\mathrm{L}_{2}$ ), presque métacentriques, sans constriction secondaire. 
Les hétérosomes se différencient en un chromosome $\mathrm{X}$ et un chromosome $\mathrm{Y}$. La femelle est homogamétique et possède deux chromosomes $X$. Le mâle est hétérogamétique et possède un chromosome $\mathrm{X}$ et un chromosome $\mathrm{Y}$.

Le chromosome $\mathrm{X}$ est sensiblement de même longueur ou légèrement plus long que l'autosome $\mathrm{L}_{1}$, mais il est nettement métacentrique et ne possède pas de constriction secondaire.

Le chromosome $\mathrm{Y}$ a, à peu près, les mêmes dimensions que l'autosome $\mathrm{L}_{2}$; il est métacentrique, comme le chromosome $\mathrm{X}$, mais il est hétérochromatique alors que les autosomes et le chromosome $\mathrm{X}$ sont euchromatiques.

La formule chromosomique de $G$. palpalis gambiensis peut donc s'écrire de la façon suivante :

Femelle: $2 \mathrm{n}=2 \mathrm{~L}_{1}+2 \mathrm{~L}_{2}+\mathrm{XX}$

Mâle : $\quad 2 \mathrm{n}=2 \mathrm{~L}_{1}+2 \mathrm{~L}_{2}+\mathrm{XY}$

\section{CARYOTYPES DES ESPECES DU SOUS-GENRE NEMORHINA (GROUPE $P A L P A L I S$ )}

Toutes les espèces étudiées dans ce sousgenre (G. fuscipes fuscipes, G. tachinoides, G. palpalis gambiensis, G. palpalis palpalis), possèdent six chromosomes, répartis en trois paires, à savoir deux paires d'autosomes $\left(\mathrm{L}_{1}\right.$ et $\mathrm{L}_{2}$ ) et une paire de chromosomes sexuels. Les espèces appartenant à ce sous-genre ont donc une formule chromosomique identique $(2 \mathrm{n}=$ 4 autosomes +2 chromosomes sexuels).

Les dimensions et la morphologie générale des autosomes et du chromosome $\mathrm{X}$ sont très semblables d'une espèce à l'autre et correspondent à la description qui a été donnée ci-dessus pour les chromosomes de $G$. palpalis gambiensis.

Seul le chromosome $\mathrm{Y}$ présente quelques différences notables suivant les espèces.

Chez $G$. fuscipes fuscipes, il est plus court que l'autosome $L_{1}$, mais plus long que l'autosome $\mathrm{L}_{2}$. II est, de plus, très nettement hétérobrachial, le bras le plus court ne faisant environ que le $1 / 5$ de la longueur totale du chromosome.

Chez G. tachinoides, G. palpalis gambiensis, $G$. palpalis palpalis, Ie chromosome $\mathrm{Y}$ est métacentrique ou submétacentrique, ses deux bras étant de longueur sensiblement égale. Il est plus court que l'autosome $\mathrm{L}_{2}$ chez $G$. tachinoides, alors que chez G. palpalis gambiensis il est légèrement plus long que Iui.

\section{CARYOTYPES DES ESPECES DU SOUS-GENRE GLOSSINA (GROUPE MORSITANS)}

Chez les espèces de ce sous-genre, on retrouve quatre autosomes $\left(2 \mathrm{~L}_{1}+2 \mathrm{~L}_{2}\right)$, semblables à ceux décrits chez les espèces du groupe palpalis. On note également, chez la femelle, deux chromosomes $\mathrm{X}$, métacentriques, ayant à peu près les mêmes dimensions que l'autosome $L_{1}$, mais ne présentant pas de constriction secondaire.

Le mâle possède un chromosome $\mathrm{X}$ et un chromosome Y. Celui-ci, toujours hétérochromatique, est, chez $G$. morsitans morsitans, très semblable au chromosome $\mathrm{Y}$ de $G$. fuscipes fuscipes, alors que le chromosome $\mathrm{Y}$ de $G$. austeni, de $G$. morsitans submorsitans et de $G$. morsitans centralis est comparable au chromosome $\mathrm{Y}$ de $G$. tachinoides, de $G$. palpalis gambiensis et de $G$. palpalis palpalis.

On trouve en outre, chez toutes les espèces du sous-genre Glossina, un jeu de petits chromosomes hétérochromatiques et télocentriques, dont le nombre varie d'une espèce à l'autre. A l'intérieur d'une même espèce ou sous-espèce, on trouve fréquemment des variations individuelles, en plus ou en moins, dans le nombre de ces petits chromosomes, autour d'une moyenne commune à l'espèce ou sous-espèce considérée.

Ces petits chromosomes, ou chromosomes $\mathrm{S}$, que dès 1970 (3) nous pensions être des chromosomes surnuméraires, ou chromosomes B, ne se retrouvent pas chez les espèces appartenant au groupe palpalis.

Les formules chromosomiques des espèces étudiées dans le groupe morsitans peuvent en conséquence s'écrire comme suit :

G. m. morsitans :

$$
\begin{gathered}
2 \mathrm{n}=2 \mathrm{~L}_{1}+2 \mathrm{~L}_{2}+\mathrm{XX} \text { (ou } \mathrm{XY} \text { ) } \\
+4( \pm 1 \text { ou } 2) \mathrm{S}
\end{gathered}
$$

G. $m$. submorsitans :

$$
\begin{gathered}
2 \mathrm{n}=2 \mathrm{~L}_{1}+2 \mathrm{~L}_{2}+\mathrm{XX} \text { (ou } \mathrm{XY} \text { ) } \\
+6( \pm 1) \mathrm{S}
\end{gathered}
$$


G. austeni :

$$
\begin{gathered}
2 \mathrm{n}=2 \mathrm{~L}_{1}+2 \mathrm{~L}_{2}+\mathrm{XX}(\mathrm{ou} \mathrm{XY}) \\
+10( \pm 2) \mathrm{S}
\end{gathered}
$$

G. $m$. centralis :

$$
\begin{gathered}
2 \mathrm{n}=2 \mathrm{~L}_{1}+2 \mathrm{~L}_{2}+\mathrm{XX} \text { (ou XY) } \\
+2 \mathrm{M}_{3}
\end{gathered}
$$

Chez cette dernière espèce, SOUTHERN (7) note la présence de deux petits chromosomes métacentriques $\left(\mathrm{M}_{3}\right)$ et l'absence de chromosomes $\mathrm{S}$ télocentriques.

D'une manière générale, la formule chromosomique des espèces appartenant au groupe morsitans serait du type:

$2 \mathrm{n}=4$ autosomes +2 chromosomes sexuels

$+\mathbf{n}$ petits chromosomes surnuméraires

\section{CONCLUSIONS}

La différence essentielle que l'on note entre les espèces appartenant au groupe morsitans et celles appartenant au groupe palpalis réside dans la présence, chez les espèces du groupe morsitans, d'un nombre variable de petits chromosomes surnuméraires qui sont totalement absents chez les espèces du groupe palpalis.

La présence de ces petits chromosomes chez les espèces du groupe morsitans traduit peutêtre une adaptation aux conditions écologiques particulières à ces espèces.

Il est en outre remarquable que des espèces aussi différentes que $G$. fuscipes fuscipes et G. morsitans morsitans possèdent, chez le mâle, des chromosomes $\mathbf{Y}$ très comparables, tandis que, de ce point de vue, G. austeni, G. morsitans submorsitans et $G$. morsitans centralis se rapprochent de $G$. tachinoides et des deux sousespèces de $G$. palpalis.

Il serait très intéressant d'étudier le caryotype des autres sous-espèces de $G$. fuscipes ( $G$. fuscipes quanzensis et $G$. fuscipes martinii), afin de vérifier si leur chromosome $Y$ est semblable à celui de $G$. fuscipes fuscipes.

\section{SUMMARY}

Caryotype of Glossina palpalis gambiensis Vanderplank, 1949. Comparison with other species of palpalis and morsitans group

Like other species of the sub-genus Nemorhina (palpalis group), Glossina palpalis gambiensis has four autosomes $\left(2 \mathrm{~L}_{1}+2 \mathrm{~L}_{2}\right)$ and two seX chromosomes (XX or XY). The species of the sub-genus Glossina (morsitans group) have, besides, a set of small supernumary heterochromatic chromosomes which vary in number according to species or subspecies.

The eu-chromosomes $\left(L_{1}, L_{2}, X\right)$ are very similar within these two sub-genus. On the contrary, the G.f. fuscipes and G.m. morsitans heterochromatic $\mathbf{Y}$ chromosome is almost acrocentric, while it is metacentric in G.p. gambiensis, G.p. palpalis, G. tachinoides, G. austeni, G. m. submorsitans and G. m. centralis.

\section{RESUMEN}

\section{Cariotipo de Glossina palpalis gambiensis Vanderplank, 1949. Comparación con otras especies del grupo palpalis $y$ del grupo morsitans}

Glossina palpalis gambiensis posec, como las otras especies del subgénero Nemorhina (grupo palpalis), quatro autosomos $\left(2 \mathrm{~L}_{1}+2 \mathrm{~L}_{3}\right)$ y dos cromosomos sexuales (XX o XY). Además, las especies del subgénero Glossina (grupo morsitans) tienen un juego de pequeños cromosomos supernumerarios, heterocromáticos, cuyo número varia de una especie o subespecie a otra.

Los eucromosomos $\left(L_{1}, L_{2}, X\right)$ son muy semejantes en los dos subgéneros.

En cambio, el cromosomo $Y$, heterocromático, es casi acrocentrico en G.f. fuscipes y $G . m$. morsitans, mientras que es metacentrico en G. p. gambiensis, G.p. palpalis, G. tachinoides, $G$. austeni, $G . m$. submorsitans y G.m. centralis. 


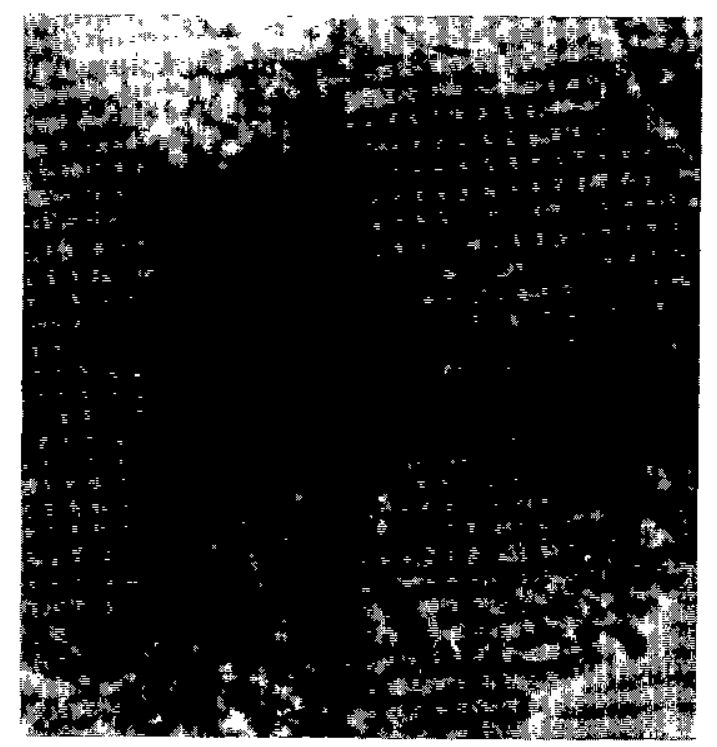

Fig. 1. - G. palpalis gambiensis femelle. Métaphase dans une cellule nerveuse (pupe de 8 jours).

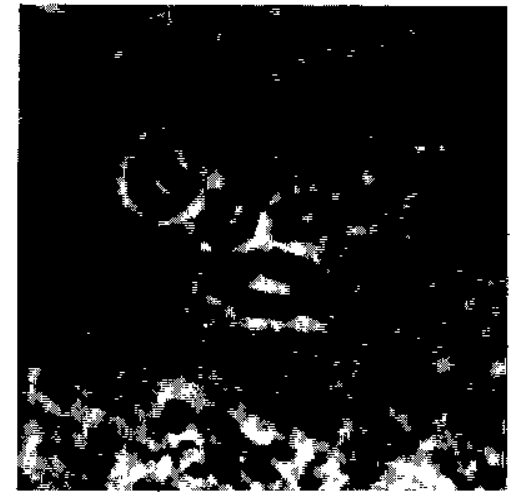

Fig. 2. - G. palpalis gambiensis mâle. Métaphase dans une cellule nerveuse (pupe de 9 jours).

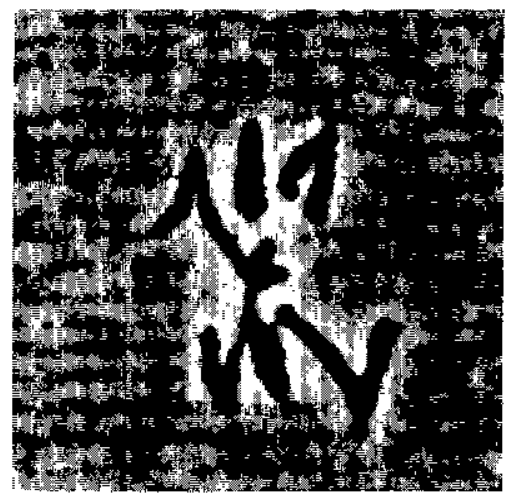

Fig. 3. - G. palpalis gambiensis. Spermatocyte d'une pupe de 8 jours. Début de l'anaphase I. 


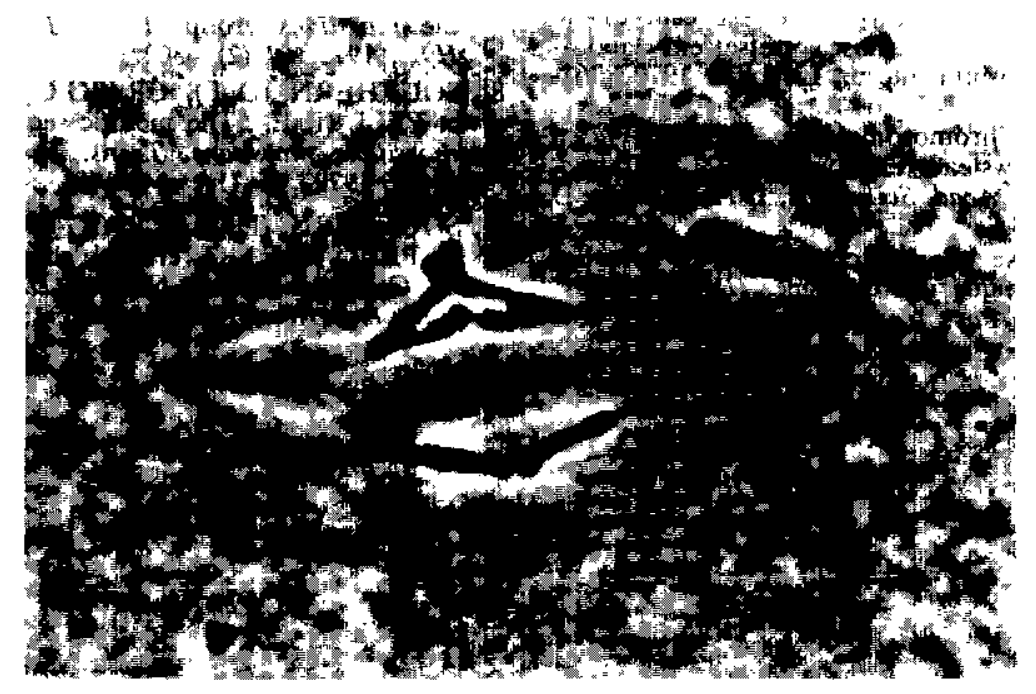

Fig. 4.

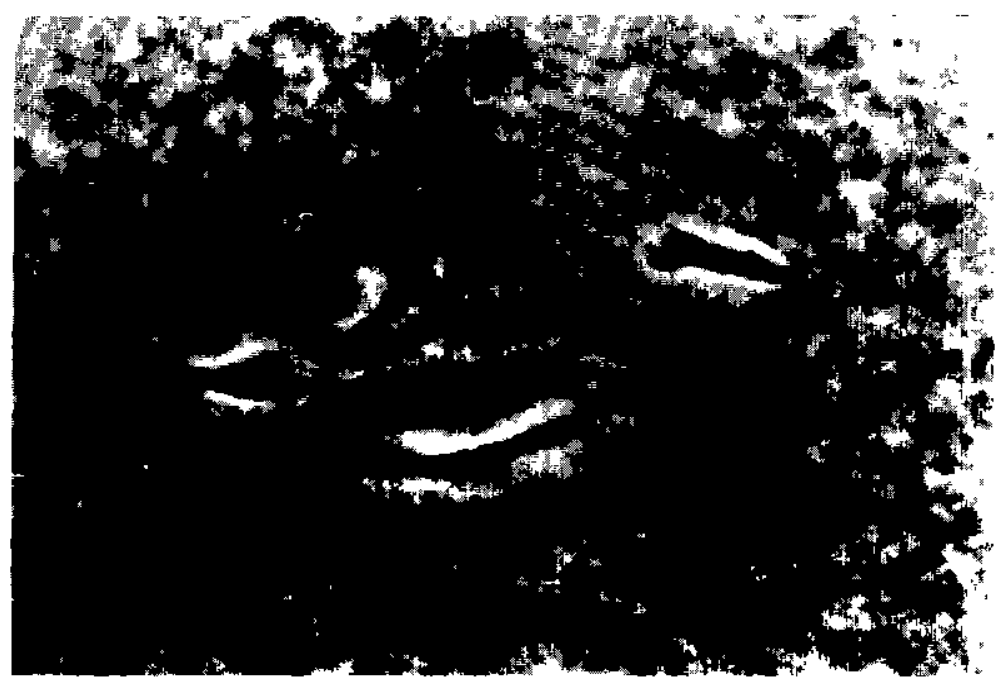

Fig. 4 et 5. - G. palpalis gambiensis. Spermatocyte d'une pupe de 8 jours. Anaphase I. Figure 4: autosomes $\mathrm{L}_{1}$ et $\mathrm{L}_{\text {q. }}$ - - Figure 5 : hétérosomes $\mathrm{X}$ et $\mathrm{Y}$. 


\section{BIBLIOGRAPHIE}

1. ITARD (J.). Chromosomes de Glossines (DipteraMuscidae). C.R. Acad. Sci. Paris, 1966, 263 Série D (19) : 1395-1397.

2. ITARD (J.). Observations sur les caryotypes de quatre espèces de glossines. I.S.C.T.R. 12e réunion. Bangui, R.C.A., novembre 1968.

3. ITARD (J.). Les caryotypes de six espèces de Glossines. Criação de mosca tsetse em laboratorio, 1er Symposium intern., Lisbonne 1970: 361367.

4. ITARD (J.). Chromosomes de Glossina fusca congolensis Newstead et Evans, 1921 (DipteraMuscidae). C.R. Acad. Sci., Paris, 1971, 272 série D (20) : 2561-2564.

5. ITARD (J.). Revue des connaissances actuelles sur la cytogénétique des Glossines (Diptera). Rev. Elev. Mèd. vét. Pays trop., 1973, 26 (2) : 151-167.
6. PELL (P. E.) et SOUTHERN (D. I.). Comparative cytogenetics of certain members of the palpalis group. Trans. R. Soc. trop. Med. Hyg., 14e Seminar on Trypanosomiasis (sous presse).

7. PELL (P.E.), SOUTHERN (D. I.) et CRAIGCAMERON (T.A.). Comparative cytogenetics of the morsitans group. Trans. R. Soc. trop. Med. Hyg., 1973, 67 (2) : 302.

8. SOUTHERN (D. I.), CRAIG-CAMERON (T. A.) et PELL (P. E.). The meiotic sequence in Glossina morsitans morsitans. Trans. $R$. Soc. trop. Med. Hyg., 1972, 66 (1) : 145-149.

9. SOUTHERN (D. I.), CRAIG-CAMERON (T. A.) et PELL (P. E.). A critical chromosome analysis of Glossina austeni Newst. (Dipt., Glossinidae). Bull. ent, Res,, 1972, 62 (2): 195-198. 